\title{
Caracterización de las propiedades tensiles de un material compuesto laminado Epoxi-Carbono empleado en la fabricación de un monoplaza tipo Fórmula SAE
}

\author{
(Characterization of the tensile properties of an Epoxy-Carbon \\ laminated composite used in the development of a single-seater \\ Formula SAE type)
}

\author{
Jorge I. Fajardo ${ }^{1}$, Micaela N. Villa², Jonatan A. Pozo ${ }^{3}$, Diego R. Urgilés ${ }^{4}$
}

\begin{abstract}
Resumen
El presente artículo analiza las propiedades tensiles de un material compuesto polimérico reforzado con fibras de carbono empleado en la fabricación del monoplaza para competición Fórmula SAE. Para ello se manufacturaron compuestos a partir de preimpregnados con diferentes orientaciones de fibra mediante un proceso de curado al vacío. Se evaluó el efecto de la orientación de las fibras sobre la resistencia y la rigidez de los compuestos formulados. Se instrumentaron las probetas de tracción con extensómetros biaxiales para obtener un registro real de la deformación y posterior determinación del módulo de Young. Los resultados experimentales fueron comparados con los resultados analíticos obtenidos a partir de la Teoría Clásica de Laminados mediante software especializado. Se desarrolló también un análisis microscópico de la región fracturada de las probetas para evaluar el efecto del proceso de manufactura. El conjunto de información generada a partir de los resultados experimentales y analíticos proporciona un entendimiento de la anisotropía de los materiales compuestos Epoxi-Carbono que permitirá tomar decisiones de diseño en futuros desarrollos dentro del proyecto Fórmula SAE.
\end{abstract}

\section{Palabras clave}

Fibras de carbono; preimpregnado; material compuesto, anisotropía; Fórmula SAE.

\begin{abstract}
This article presents the analysis of the tensile properties of a carbon fiber reinforced polymer composite used in the manufacture of the single-seater for Formula SAE competition. Composites were manufactured from pre-pregs with different fiber orientations by a vacuum curing process. The effect of the fiber orientation on the strength and rigidity of the formulated composites was evaluated. The tensile specimens were instrumented with biaxial extensometers in order to obtain a real record of the deformation and subsequent determination of the Young's modulus. The experimental results were compared with the analytical results obtained from the Classical Laminate Theory using specialized software. A microscopic analysis of the fractured region of the specimens was also developed to evaluate the effect of the manufacturing process. The set of information generated from the experimental and analytical results provides an understanding of the anisotropy of Epoxy-Carbon fiber composites that will allow design decisions to be made in future developments within the Formula SAE project.
\end{abstract}

\section{Keywords}

Carbon fiber; pre-preg; composite; anisotropy, Formula SAE.

\section{Introducción}

Los laminados son materiales compuestos reforzados con fibras y son ampliamente utilizados en aplicaciones estructurales que requieren una combinación de resistencia y bajo peso; dentro

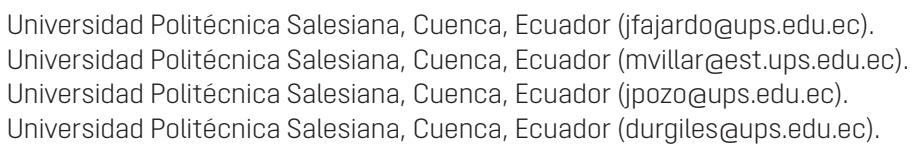


de estas aplicaciones se incluyen las industrias: aeronáutica, marina, automotriz e infraestructura (Krishnamoorthy y Sasikumar, 2016; Mallick, 2007; Noroña y Gómez, 2019; Paredes, Pérez, y Castro, 2017). Cuando se utilizan tejidos en materiales compuestos es común encontrar características como integridad, conformabilidad y propiedades equilibradas (Murugan, Ramesh, y Padmanabhan, 2014). Cuando el reforzante es sintético, como el caso de las fibras de carbono, se encuentran ventajas importantes como: alta resistencia específica a tracción, resistencia a la fatiga y alta conductividad térmica (Mallick, 2007). Por otra parte, la principal desventaja de los materiales compuestos reforzados con fibra continua suele ser su elevada anisotropía, lo que dificulta el estudio analítico y el diseño de componentes. Sin embargo, al realizar pruebas estandarizadas es posible determinar el comportamiento del material y con una caracterización mecánica adecuada es posible entender el comportamiento mecánico del material que permita realizar diseños adecuados (Al-Mosawi, 2014; Liu et al., 2017; Schulenberg, Seelig, Andrieux, y Sun, 2017; Viscardi, Arena, Barra, y Guadagno, 2016; Yaqiang, Xin, y Zhishen, 2017).

Utilizar materiales compuestos mediante técnicas de laminado es muy práctico, ya que permite el uso de estructuras de paredes finas a partir del uso de pre-impregnados (Elkington, Bloom, Ward, Chatzimichali, y Potter, 2015). En la Tabla 1 se resumen los resultados que presenta la literatura revisada sobre procesos de laminado manual en compuestos poliméricos epoxicarbono, con curados en autoclave.

Tabla 1. Propiedades mecánicas de materiales compuestos laminados epoxi-carbono

\begin{tabular}{|c|c|c|c|c|c|c|c|c|c|c|}
\hline Reforzante & $V_{f}$ & Espesor & $\begin{array}{l}\mathrm{N}^{\circ} \text { de } \\
\text { capas }\end{array}$ & $\mathrm{T}^{\circ} \mathrm{C}$ & $\begin{array}{l}\text { Presión de } \\
\text { curado }\end{array}$ & $\begin{array}{l}\text { Tipo de } \\
\text { curado }\end{array}$ & $\begin{array}{l}\text { Esfuerzo a } \\
\text { tracción }\end{array}$ & $\begin{array}{l}\text { Defor. a } \\
\text { rotura }\end{array}$ & $\begin{array}{l}\text { Módulo de } \\
\text { Young }\end{array}$ & Referencia \\
\hline Prepreg UD & 0,593 & 1 & 10 & 130 & 245.17 & 0.83 & 2428 & $\approx 0.015$ & 133.8 & $\begin{array}{l}\text { (Ma, Yang, Sugahara, y } \\
\text { Hamada, 2016) }\end{array}$ \\
\hline Prepreg UD & N/A & N/A & 8 & N/A & N/A & N/A & $\approx 1850$ & N/A & N/A & $\begin{array}{l}\text { (Majerski, Surowska, y } \\
\text { Bieniaś, 2012) }\end{array}$ \\
\hline Tejido satín & N/A & $\mathrm{N} / \mathrm{A}$ & N/A & 20 & 500 & 168 & 370 & 0.26 & 142 & $\begin{array}{l}\text { (Ramana y Rampra- } \\
\text { sad, 2017) }\end{array}$ \\
\hline Prepreg UD & N/A & 0.125 & $\mathrm{~N} / \mathrm{A}$ & 170 & N/A & N/A & 1700 & 1.16 & 127 & $\begin{array}{l}\text { (Bailey, Curtis, y Parvi- } \\
\text { zi, 1979) }\end{array}$ \\
\hline Prepreg UD & N/A & 0,125 & N/A & 170 & N/A & N/A & 39 & 0.48 & 8.3 & (Bailey et al., 1979) \\
\hline Prepreg BA & $\mathrm{N} / \mathrm{A}$ & $2-3$ & N/A & 177 & 80 & 2 & 1185.4 & $\mathrm{~N} / \mathrm{A}$ & 65.6 & (Paiva et al., 2006) \\
\hline Prepreg 8HS & N/A & $2-3$ & N/A & 121 & 80 & 1.5 & 810 & $\mathrm{~N} / \mathrm{A}$ & 67.8 & (Paiva et al., 2006) \\
\hline Prepreg 8HS & N/A & $2-3$ & N/A & 177 & 80 & 2 & 985.9 & N/A & 71.5 & (Paiva et al., 2006) \\
\hline Prepreg UD & $\approx 0.475$ & 1 & 10 & 130 & 2451.66 & 0.83 & 1664 & $\approx 0.0145$ & 109.1 & (Ma et al., 2017) \\
\hline Tejido & 0.4 & 2.49 & 4 & 20 & 2500 & 24 & 462.08 & $\approx 0.096$ & N/A & (Murugan et al., 2014) \\
\hline Tejido & 0.4 & 2.38 & 4 & 20 & 2500 & 24 & 413.62 & $\approx 0.076$ & N/A & (Murugan et al., 2014) \\
\hline Tejido & 0.4 & 2.38 & 4 & 20 & 2500 & 24 & 403.41 & $\approx 0.068$ & N/A & (Murugan et al., 2014) \\
\hline
\end{tabular}

Prepreg: pre-impregnado

UD: unidireccional

BA: biaxial

Defor. a la rotura: deformación a la rotura

$V_{f}$ : contenido de fibra

$\mathrm{T}^{\circ} \mathrm{C}$ : temperature de curado 
El proceso de manufactura utilizado para la laminación influye sobre las propiedades mecánicas del producto final. El laminado manual se aplica en productos de diferentes tamaños y disposiciones de fibra (planar, orientada, corta o continua). Presenta como desventajas, tiempos de curado altos y de mediana calidad en acabados (Elkington et al., 2015; Mcllhagger, Archer, y Mcllhagger, 2015). Un material compuesto laminado puede estar formado por varias capas con diferentes orientaciones. Este efecto multicapa puede generar bajas propiedades interlaminares y debilitar al compuesto por delaminación. La delaminación puede ocurrir durante la fabricación, presentándose como poros o discontinuidades, también puede producirse por eventos externos como tensiones residuales generadas por la humedad, la temperatura, etc. De igual manera, la delaminación para todos los casos podría causar una falla prematura del material (Aveiga y Ribeiro, 2018; Beaumont, Soutis, y Hodzic, 2017; Chermoshentseva, Pokrovskiy, y Bokhoeva, 2016; Mehta y Vadher, 2017; Wang et al., 2019).

En la Universidad Politécnica Salesiana (UPS), desde el año 2011, el grupo de Investigación en Nuevos Materiales y Procesos de Transformación (GiMaT) ha desarrollado trabajos sobre materiales compuestos (Albarracín Parra y Castillo Agurto, 2014; Fajardo, Valarezo, López, y Sarmiento, 2013; López, Sarmiento, Fajardo, Valarezo, y Zuluaga, s. f.; Paltán, 2016). Por otra parte, el Grupo de Investigación en Ingeniería de Transporte (GIIT), de la UPS, ha venido trabajando en el proyecto FSAE, manufacturando componentes estructurales ligeros para la construcción de un monoplaza de competición para FSAE, gestionado por el Laboratorio de Aplicación de Materiales en Ingeniería del Transporte (LabAMiT). Se han utilizado refuerzos de fibra de vidrio, carbono y naturales (Espinoza e Hidalgo, 2016); A pesar del importante desarrollo en la manufactura y ensamble de componentes de monoplazas para competición FSAE, se desconocen las propiedades mecánicas del material a base de su configuración y no se dispone de registros de sus propiedades tensiles que sirvan como referente para futuros diseños en componentes similares, lo que limita sus aplicaciones. Dentro de los componentes manufacturados con preimpregnado de fibra de carbono, por el grupo FSAE de la UPS, se encuentran: el volante, el tablero, la pedalera y el fondo plano, algunos de estos se muestran en la Figura 1.

Figura 1. Componentes del vehículo FSAE UPS fabricados con preimpregndado de fibra de carbono

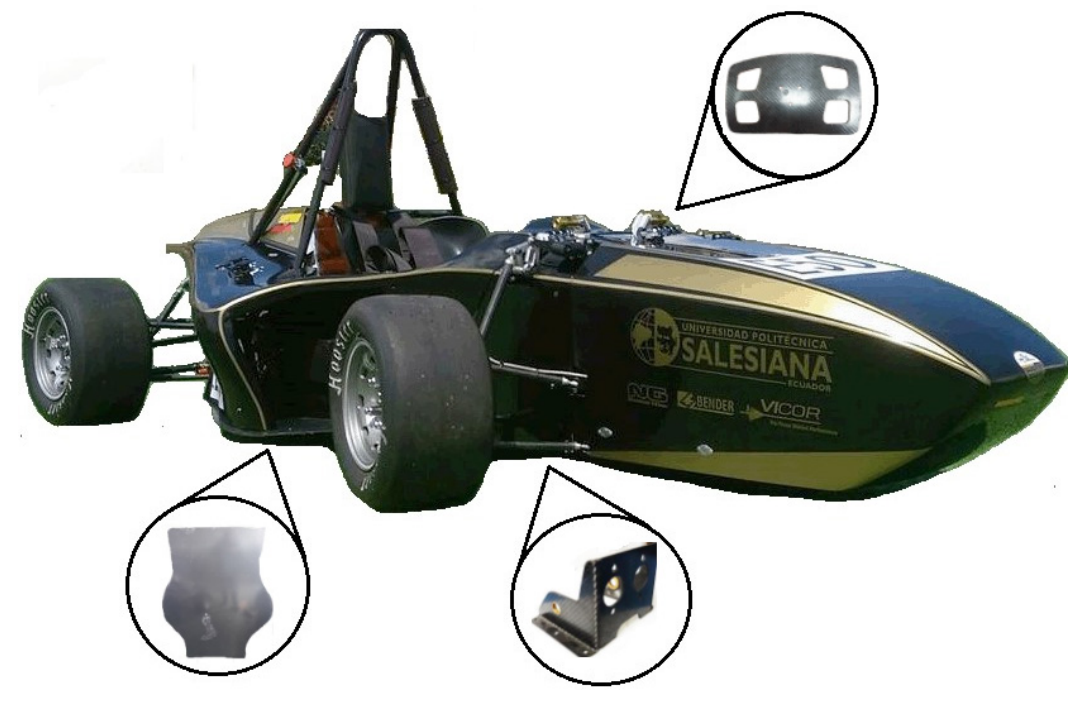


En el presente trabajo se describe el proceso de manufactura para la elaboración de probetas para ensayos de tracción unidimensional en compuestos Epoxi-Carbono con diferentes estados de orientación. Se reportan también las propiedades tensiles de los compuestos desarrollados y se analiza su anisotropía con miras a una optimización del patrón de laminado empleado en la fabricación de componentes del vehículo de competencia FSAE.

\section{Metodología}

\subsection{Materiales}

Se utilizó un preimpregnado Epoxi-Carbono en forma de tejido biaxial $2 \times 23 \mathrm{~K}$ proporcionado por ACP Composites.

\subsection{Proceso de manufactura}

Fueron fabricadas 20 probetas clasificadas de acuerdo con la secuencia de apilamiento mostrada en la Tabla 2. Estas arquitecturas fueron escogidas con la finalidad de obtener laminados balanceados y simétricos (similares a los actualmente manufacturados en el LabAMiT).

Tabla 2. Distribución de probetas de acuerdo con la secuencia de apilado

\begin{tabular}{|c|c|c|}
\hline $\begin{array}{l}\text { Cantidad } \\
\text { de probetas }\end{array}$ & Secuencia de apilamiento & $\begin{array}{l}\text { Designación } \\
\text { en el ensayo }\end{array}$ \\
\hline 5 & {$\left[(0 / 90)_{11}\right]_{\mathrm{T}}$} & $1-1,1-2,1-3,1-4,1-5$ \\
\hline 5 & {$\left[( \pm 45)_{11}\right]_{T}$} & $2-1,2-2,2-3,2-4,2-5$ \\
\hline 5 & {$\left[(30 /-60)_{11}\right]_{\mathrm{T}}$} & $3-1,3-2,3-3,3-4,3-5$ \\
\hline 5 & {$\left[(0 / 90)_{2^{\prime}} \pm 45,(0 / 90), \pm 45,(0 / 90), \pm 45,(0 / 90), \pm 45,(0 / 90)_{2}\right]_{T}$} & $4-1,4-2,4-3,4-4,4-5$ \\
\hline
\end{tabular}

El proceso de fabricación fue laminado manual y curado con temperatura al vacío. El esquema de preparación al vacío se muestra en la Figura 2. El proceso de curado inició a temperatura ambiente y alcanzó una temperatura de $154^{\circ} \mathrm{C}$ a una velocidad de $1000{ }^{\circ} \mathrm{C} / \mathrm{h}$ durante 1.5 horas para luego disminuir gradualmente a temperatura ambiente. Todo el proceso se realizó a presión constante de $70 \mathrm{KPa}$. Las dimensiones de las probetas fueron 2.5×25×250 mm³ y están de acuerdo con la Norma ASTM D3039.

Figura 2. Esquema de preparación al vacío

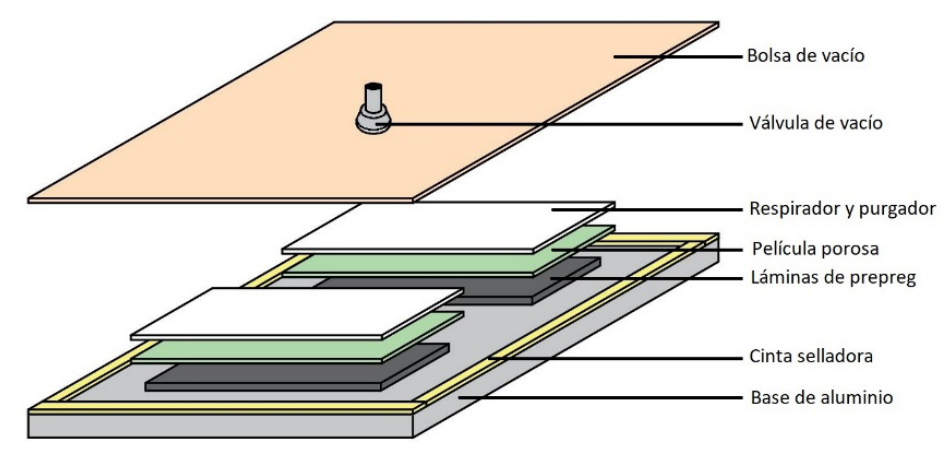




\subsection{Ensayos tensiles}

Las propiedades tensiles de las probetas fueron analizadas de acuerdo con ASTM D3039. Se utilizó una máquina universal TestResources modelo 315 Family Universal Test Machine. Además se instrumentaron extensómetros biaxiales marca Epsilon modelo SN E98328. Las propiedades caracterizadas fueron esfuerzo máximo, Módulo de Young y coeficiente de Poisson. La Figura 3 muestra el montaje e instrumentación de las probetas.

Figura 3. Montaje e instrumentación de probetas

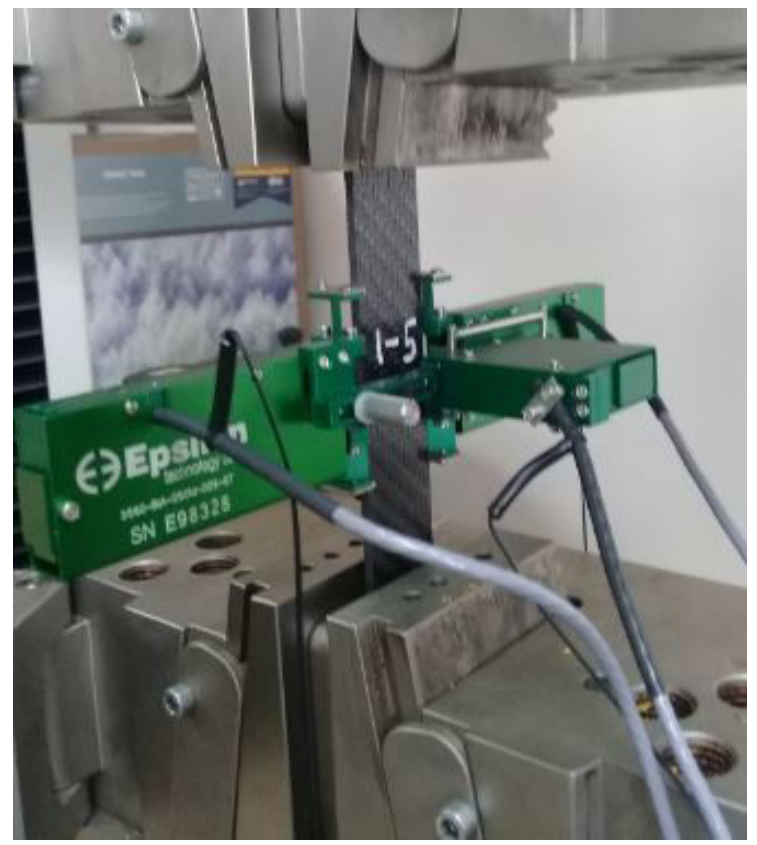

\section{Resultados}

\subsection{Modos de fallo}

La Norma ASTM D3039 detalla los fallos típicos que pueden presentarse en las probetas después de ser ensayadas. Algunas zonas de fractura típicas producidas en las probetas ensayadas son presentadas en la Figura 4 y los resultados del tipo de fallo para todas las probetas se muestran en la Tabla 3. Con base en la norma; los tipos de fallo encontrados en las distintas configuraciones de los laminados fueron: LAT fallo lateral en la mordaza y parte superior de la probeta, LMV fallo lateral en múltiples áreas de la probeta, AGM fallo angular en el área de calibración de la probeta, DGM delaminación en el borde del área de calibración, finalmente LGM fallo lateral en el área de calibración de la probeta. La Norma reconoce que pueden existir otros fallos no mencionado en la misma que deben ser reportados en el informe. 
Figura 4. Probetas después de los ensayos: (a) probeta 1-2 (LMV), (b) probeta 2-3 (AGM), (c) probeta 3-3 (DGM), (d) probeta 4-4 (LAT)

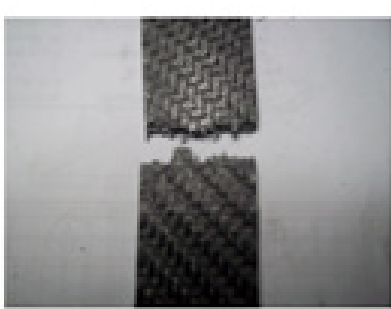

(a)

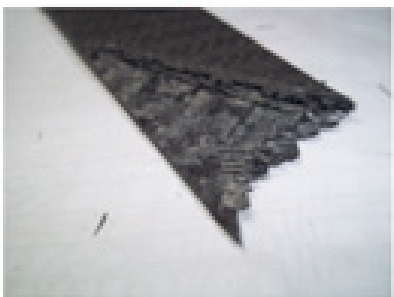

(c)

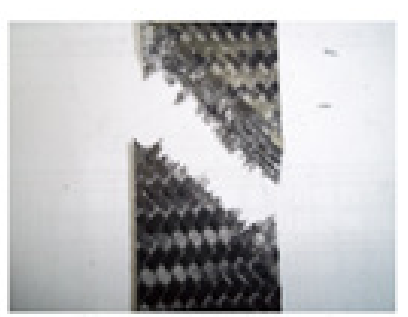

(b)

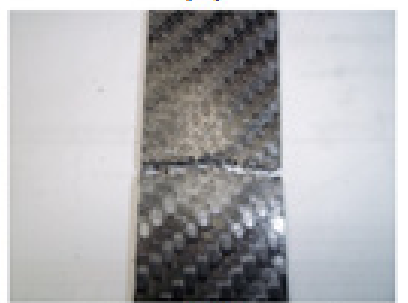

(d)

Tabla 3: Tipo de fallo en las probetas

\begin{tabular}{|c|c|c|c|c|c|}
\hline Probeta & Laminado & $\begin{array}{l}\text { Tipo } \\
\text { de fallo }\end{array}$ & Probeta & Laminado & $\begin{array}{l}\text { Tipo } \\
\text { de fallo }\end{array}$ \\
\hline $1-1$ & \multirow{5}{*}[(0/90)_{11}]{$_{\mathrm{T}}$} & LAT & $3-1$ & \multirow{5}{*}[(30/-60)_{11}]{$_{T}$} & DGM \\
\hline $1-2$ & & LMV & $3-2$ & & DGM \\
\hline $1-3$ & & LAT & $3-3$ & & DGM \\
\hline $1-4$ & & LAT & $3-4$ & & LGM \\
\hline $1-5$ & & LAT & $3-5$ & & LGM \\
\hline $2-1$ & \multirow{5}{*}[(\pm45)_{11}]{$_{\mathrm{T}}$} & N/A & $4-1$ & \multirow{5}{*}{$\begin{array}{l}(0 / 90)_{2^{\prime}} \pm 45,(0 / 90), \pm 45,(0 / 90) \\
\left.\quad \pm 45,(0 / 90), \pm 45,(0 / 90)_{2}\right]_{\top}\end{array}$} & LAT \\
\hline $2-2$ & & AGM (2) & $4-2$ & & LAT \\
\hline $2-3$ & & AGM (2) & $4-3$ & & LAT \\
\hline $2-4$ & & N/A & 4-4 & & LAT \\
\hline $2-5$ & & N/A & $4-5$ & & LAT \\
\hline
\end{tabular}

\subsection{Propiedades tensiles de los compuestos}

La Figura 5 resume los valores promedios y la desviación estándar de las propiedades tensiles evaluadas para cada configuración del material compuesto. 
Figura 5. Efecto de la orientación de los laminados sobre las propiedades tensiles de compuestos Epoxi-Carbono

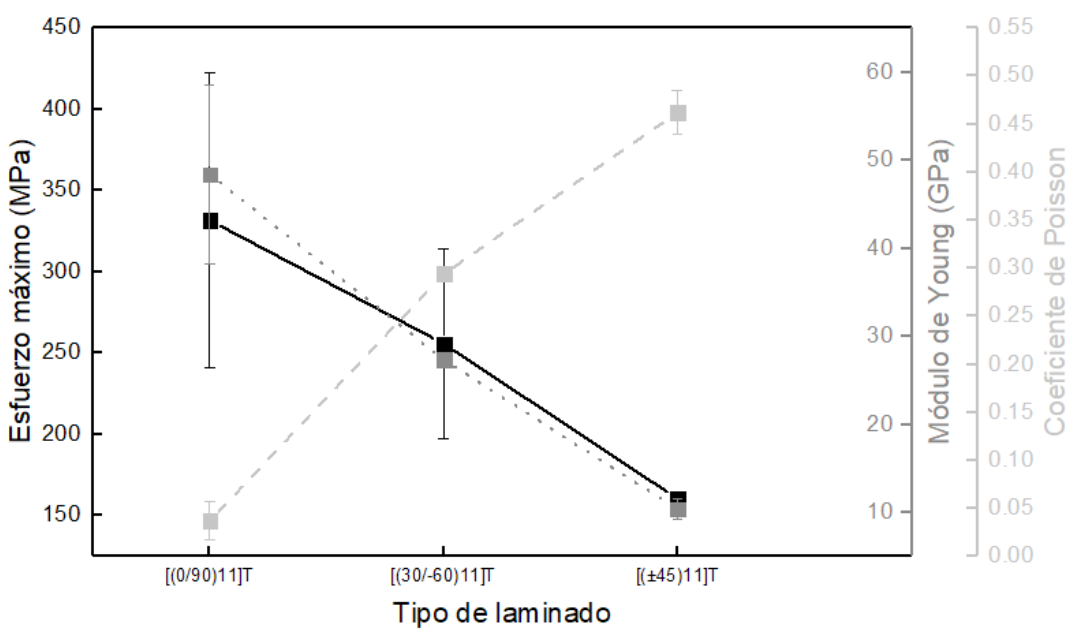

Como se observa en la Figura 5, a medida que se incrementa el ángulo de orientación con respecto a la dirección de la carga tensil, la resistencia a tracción y el módulo de Young de los compuestos disminuyen progresivamente mientras que el coeficiente de Poisson se incrementa, es decir, existe una relación inversa entre estos parámetros. De acuerdo con la literatura para un pre-impregnado biaxial se pueden alcanzar, en promedio, valores de $1185.4 \mathrm{MPa}$ y de 65.6 GPa para el esfuerzo máximo y el módulo de Young respectivamente (Paiva et al., 2006). La diferencia entre los valores obtenidos en esta investigación y los reportados en la literatura se deben a errores sistemáticos y a la presencia de defectos en la manufactura.

Con la finalidad de comprender mejor los efectos del proceso de manufactura sobre las propiedades de las probetas desarrolladas, se realizó una inspección microscópica en la zona de fractura del compuesto. A partir de este análisis se determinó que las probetas presentan delaminación. De las muestras tomadas en el microscópico, en la Figura 6 (a) se muestran porosidades $(P)$ presentes en el interior de las láminas, mientras que en la Figura 5 (b) se distingue una grieta (G) en la dirección de la carga, pero en una zona de fibras transversales del tejido. Esta observación confirma la presencia de defectos producidos durante el proceso de manufactura que requieren atención posterior.

Figura 6. Microfotografías de las probetas después del ensayo a tracción
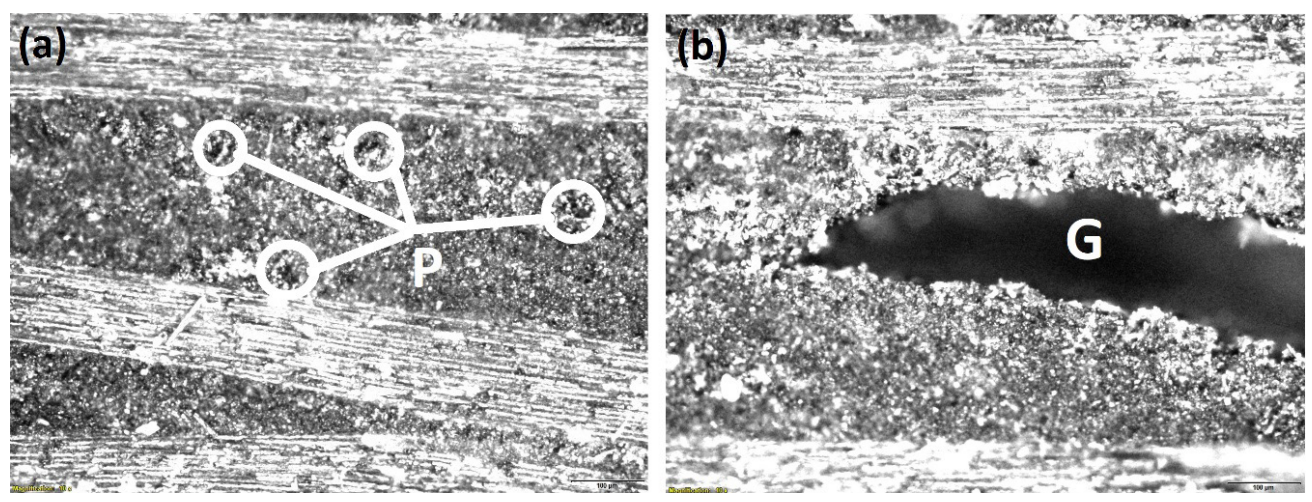
La evaluación de la anisotropía de las propiedades tensiles fue realizada mediante la construcción de cartas polares. Con base en la Figura 7, se observa un comportamiento anisotrópico en los tres laminados ensayados (Figura 7 (a), (b) y (c)). En un laminado de tipo [(0/90) $\left.{ }_{11}\right]$ se logran mayores valores para el módulo de Young cuando la carga aplicada tiene una orientación a $0^{\circ}$ y $90^{\circ}$; mientras que los valores de coeficiente de Poisson son más altos cuando la carga es aplicada en ángulos de $\pm 45^{\circ}$. Este laminado se define como anisotrópico pues solo alcanza valores máximos de las propiedades en orientaciones determinadas. Este comportamiento es similar para las configuraciones de $[(30 /-60) 11]_{\mathrm{T}}$ y $[( \pm 45) 11]_{\mathrm{T}}$. El laminado de la Figura $7(d)[(0 / 90) 2, \pm 45,(0 / 90), \pm 45,(0 / 90), \pm 45,(0 / 90), \pm 45,(0 / 90) 2]_{T^{\prime}}$ es cuasi-isotrópico debido que tiende a presentar propiedades similares independientemente de la dirección de la carga, esta característica es de gran importancia cuando se desea diseñar componentes que estarán sometidos a cargas externas en diferentes orientaciones.

Figura 7. Cartas polares de los laminados Epoxi - Carbono analizados (a) $\left[(0 / 90)_{11}\right]_{T^{\prime}}$ (b) $\left[(30 /-60)_{11}\right]_{T^{\prime}}$ (c) $\left[( \pm 45)_{11}\right]_{T^{\prime}}$ (d) $\left[(0 / 90)_{2^{\prime}} \pm 45,(0 / 90), \pm 45,(0 / 90), \pm 45,(0 / 90), \pm 45,(0 / 90)_{2}\right]_{T}$.

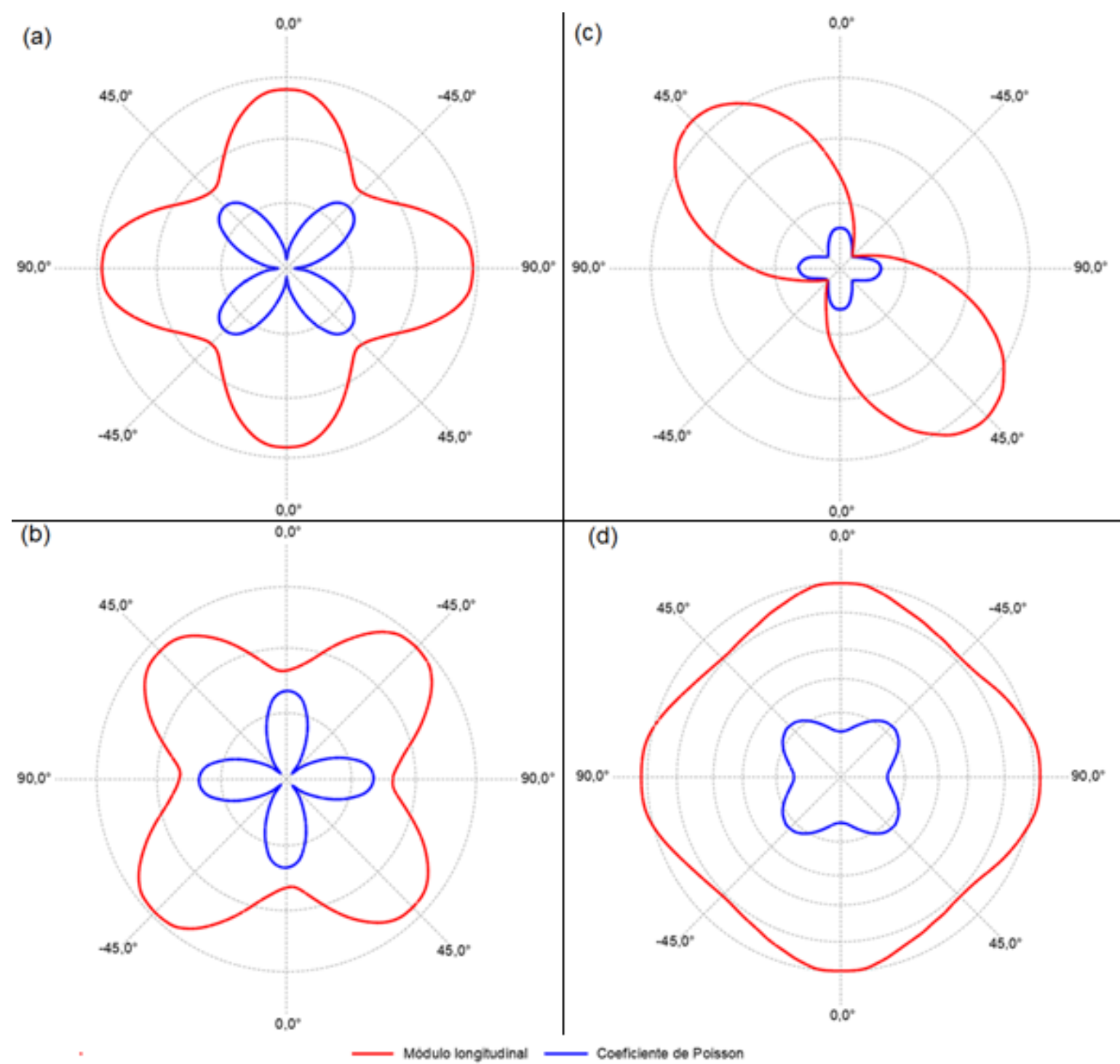


Para verificar los resultados experimentales con respecto a la Teoría Clásica de Laminados (TCL) y evaluar el proceso de manufactura, se utilizó el software eLamX (Dresden, s. f.). El programa cuenta con una base de datos de materiales compuestos. Para la validación se seleccionó el material cuyas propiedades fueron similares a las del compuesto desarrollado, C-'CFS003' | EP-'LTM25'. La comparación entre los resultados del software y los resultados experimentales promedio para las probetas se muestra en la Tabla 4.

Tabla 4. Comparación entre Resultados experimentales y analíticos

\begin{tabular}{|c|c|c|c|c|c|c|}
\hline \multirow[t]{2}{*}{ Laminado } & \multirow[t]{2}{*}{$\begin{array}{l}\text { Método } \\
\text { de análisis }\end{array}$} & $\begin{array}{l}\text { Esfuerzo } \\
\text { a tracción }\end{array}$ & $\begin{array}{l}\text { Módu- } \\
\text { lo de } \\
\text { Young }\end{array}$ & \multirow[t]{2}{*}{$v$} & $\begin{array}{l}\text { Esfuerzo } \\
\text { a tracción } \\
\text { específico }\end{array}$ & $\begin{array}{l}\text { Módulo } \\
\text { de Young } \\
\text { específico }\end{array}$ \\
\hline & & (MPa) & (GPa) & & $\left(\mathrm{MPa} / \mathrm{g} \cdot \mathrm{cm}^{-3}\right)$ & $\left(\mathrm{GPa} / \mathrm{g} \cdot \mathrm{cm}^{-3}\right)$ \\
\hline \multirow{4}{*}[(0/90)_{11}]{$_{T}$} & Experimental & 331.727 & 48.382 & 0.037 & 210.459 & 30.695 \\
\hline & CLT & 361.264 & 53.6 & 0.042 & 274.638 & 40.747 \\
\hline & Error rel. (\%) & 8.176 & 9.735 & 11.905 & 23.369 & 24.670 \\
\hline & COV & 27.272 & 20.943 & 53.739 & 27.272 & 20.943 \\
\hline \multirow{4}{*}[(30/-60)_{11}]{$_{\mathrm{T}}$} & Experimental & 255.834 & 27.328 & 0.294 & 162.310 & 17.337 \\
\hline & CLT (UD 30) & 275.892 & 27.289 & 0.15 & 209.737 & 20.745 \\
\hline & Error rel. (\%) & 7.270 & 0.143 & 96.000 & 22.613 & 16.430 \\
\hline & COV & 22.807 & 0.644 & 2.774 & 1.053 & 11.370 \\
\hline \multirow{4}{*}[(\pm45)_{11}]{$_{\mathrm{T}}$} & Experimental & 160.473 & 10.346 & 0.462 & 101.810 & 6.564 \\
\hline & CLT & 163.333 & 10,361 & 0.818 & 124.168 & 7.877 \\
\hline & Error rel. (\%) & 1.751 & 0.145 & 43.521 & 18.006 & 16.664 \\
\hline & COV & 1.053 & 11.370 & 4.923 & 22.807 & 0.644 \\
\hline \multirow{4}{*}{$\begin{array}{c}{\left[(0 / 90)_{2^{\prime}} \pm 45\right.} \\
(0 / 90), \pm 45,(0 / 90), \\
\pm 45,(0 / 90), \pm 45 \\
\left.(0 / 90)_{2}\right]_{T}\end{array}$} & Experimental & 397.286 & 48.689 & 0.135 & 252.051 & 30.890 \\
\hline & CLT & 467.81 & 48.732 & 0.129 & 355.636 & 37.047 \\
\hline & Error rel. (\%) & 15.075 & 0.088 & 4.651 & 29.127 & 16.619 \\
\hline & COV & 5,118 & 10.232 & $\mathrm{~N} / \mathrm{A}$ & 5.118 & 10.232 \\
\hline
\end{tabular}

Error rel.: error relativ.

UD: unidireccional

v: coeficiente de Poisson

\section{Discusión}

Como se observó en la Tabla 1, para compuestos poliméricos epoxy-carbono en configuraciones de tejido a $0^{\circ} / 90^{\circ}$ los valores de esfuerzo máximo oscilan entre $462 \mathrm{MPa}$ (Murugan et al., 2014) y $1185 \mathrm{MPa}$ (Paiva et al., 2006), mientras que el módulo de Young presenta valores entre 57 y 71 GPa (Paiva et al., 2006). Para configuraciones de tejido a $\pm 45^{\circ}$ los valores de esfuerzo máximo bordean los 75 MPa y el módulo de Young cerca de 5 GPa (Çeçen, Sarikanat, Yildiz, y Tavman, 2008). Los valores más altos de las propiedades tensiles corresponden a compuestos obteni- 
dos en autoclave, con elevado control de las variables de procesamiento. Las diferencias encontradas entre los resultados provistos por la literatura y las simulaciones mediante $T C L$, con los valores obtenidos experimentalmente, en el presente estudio, obedecen en gran medida al proceso de manufactura empleado, como se demostró en el análisis microscópico de los laminados. Es importante conocer las limitaciones de cada proceso de manufactura para establecer límites de validez de los resultados obtenidos en las simulaciones y establecer los factores de seguridad adecuados en la fase de diseño. Las diferencias relativas entre las simulaciones y los resultados experimentales fueron menores al $10 \%$ para casi todos los compuestos formulados (excepto en la configuración cuasi-isotrópica) al evaluar tanto su resistencia y rigidez, lo que valida el proceso de manufactura desarrollado. El coeficiente de Poisson fue la propiedad tensil que más diferencias relativas presentó entre las simulaciones y las experimentaciones. Se evidenció la anisotropía de los compuestos formulados a diferentes orientaciones de las fibras y se consiguió un comportamiento cuasi-isotrópico al diseñar un laminado apropiado para este tipo de solicitaciones mecánicas.

\section{Conclusiones y recomendaciones}

El método utilizado actualmente genera ciertos defectos volumétricos como poros y falta de adhesión entre láminas en algunos sectores del laminado que derivan en reducción de las propiedades tensiles del compuesto generando errores relativos menores al $10 \%$ con respecto a los valores esperados para estos materiales con base en los resultados provistos por la literatura y a las simulaciones empleando TCL. La caracterización de las propiedades tensiles de diferentes configuraciones de materiales compuestos Epoxy-carbono permite definir una base de datos de las propiedades de los materiales compuestos Epoxi-Carbono desarrollados en los laboratorios LabAMit de la UPS, esta información será de gran utilidad para futuros diseños dentro del proyecto FSAE. En general los compuestos formulados epoxi- carbono presentaron la mejor combinación de propiedades específicas en la configuración $\left[(0 / 90)_{11}\right]_{T}$ dado que las fibras de carbono fueron capaces de aportar la totalidad de su efecto reforzante. No obstante, se ven limitadas cuando están sometidas a cargas en direcciones dentro del intervalo: $0^{\circ}<\alpha>90^{\circ}$. Los compuestos formulados con orientación de las fibras a $\pm 45^{\circ}$ permiten que el material presente pseudo-ductilidad debido al mayor aporte que realiza la matriz Epoxi desde el inicio de la deformación hasta que las fibras tienden a realinearse. Un laminado optimizado sería aquel que combine las orientaciones antes mencionadas de acuerdo con las necesidades de rigidez o ductilidad del componente a fabricar, es decir utilizar un material con características cuasiisotrópicas. Un laminado con una configuración de $30^{\%}-60^{\circ}$ no es recomendable ya que esto genera que el laminado no sea balanceado y que el refuerzo tejido se comporte como un material unidireccional, y por lo tanto se genere una subutilización del mismo. Se demostró que es posible conseguir un comportamiento cuasi-isotrópico al diseñar un laminado con orientaciones $[(0 / 90) 2, \pm 45,(0 / 90), \pm 45,(0 / 90), \pm 45,(0 / 90), \pm 45,(0 / 90) 2]_{T}$; esta configuración es muy utilizada cuando se requiere una combinación de buenas propiedades e isotropía. La resistencia y el módulo fueron $16.5 \%$ menores al laminado $[(0 / 90) 11]_{\mathrm{T},}$ pero se consiguen propiedades similares en todas las direcciones de aplicación de la carga.

En trabajos posteriores se realizará el análisis mediante elementos finitos de un componente real de una fórmula SAE, alimentando las propiedades del material con los resultados obtenidos en el presente estudio. 


\section{Referencias}

Albarracín Parra, P., y Castillo Agurto, E. (2014). Determinación de la distribución de orientación y longitud de fibras mediante procesamiento digital de imágenes en compuestos poliméricos reforzados con fibras cortas. Tesis de grado, Universidad Politécnica Salesiana.

Al-Mosawi, A. I. (2014). Theoretical Evaluation to Tensile Strength of Composite Material by Using Ansys Program. Engineering Sciences, 4 (22).

Aveiga, D., y Ribeiro, M. L. (2018). A Delamination Propagation Model for Fiber Reinforced Laminated Composite Materials. Mathematical Problems in Engineering. https://doi.org/10.1155/2018/1861268

Bailey, J. E., Curtis, P. T., y Parvizi, A. (1979). On the transverse cracking and longitudinal splitting behaviour of glass and carbon fibre reinforced epoxy cross ply laminates and the effect of Poisson and thermally generated strain. Proc. R. Soc. Lond. A, 366 (1727): 599-623. https://doi. org/10.1098/rspa.1979.0071

Beaumont, P. W. R., Soutis, C., y Hodzic, A. (eds.). (2017). The Structural Integrity of Carbon Fiber Composites: Fifty Years of Progress and Achievement of the Science, Development, and Applications. Suiza: Springer International Publishing.

Çeçen, V., Sarikanat, M., Yildiz, H., y Tavman, I. H. (2008). Comparison of mechanical properties of epoxy composites reinforced with stitched glass and carbon fabrics: Characterization of mechanical anisotropy in composites and investigation on the interaction between fiber and epoxy matrix. Polymer Composites, 29 (8): 840-853. https://doi.org/10.1002/pc.20458

Chermoshentseva, A. S., Pokrovskiy, A. M., y Bokhoeva, L. A. (2016). The Behavior of Delaminations in Composite Materials-Experimental Results. IOP Conference Series: Materials Science and Engineering, 116. https://doi.org/10.1088/1757-899X/116/1/012005

Dresden, T. U. D. U. (s. f.). eLamX. Recuperado de https://tu-dresden.de/ing/maschinenwesen/ilr/lft/ela$\mathrm{m} \times 2 /$ startseite/?set_language $=e n$

Elkington, M., Bloom, D., Ward, C., Chatzimichali, A., y Potter, K. (2015). Hand layup: Understanding the manual process. Advanced Manufacturing: Polymer \& Composites Science, 1 (3), 138-151. https:// doi.org/10.1080/20550340.2015.1114801

Espinoza, E., y Hidalgo, J. (2016). Caracterización de materiales compuestos para la aplicación en la carrocería del vehículo monoplaza tipo Formula SAE (Master's Thesis).

Fajardo, J., Valarezo, L., López, L., y Sarmiento, A. (2013). Experiencies in obtaining polymeric composites reinforced with natural fiber from Ecuador. Ingenius. Revista de Ciencia y Tecnología 9: 27-35.

Krishnamoorthy, K., y Sasikumar, T. (2016). Analysis and Characterization of Tensile Property of the Composite Specimen using ANSYS, 11 (1): 380-384.

Liu, J. D., Yang, X. F., Xu, Z. L., Li, Y. S., Yan, G. H., y Huang, L. K. (2017). Experimental Study on the Tool Wear in Milling Carbon Fiber Reinforced Plastics. Materials Science Forum, 893, 57-61. https://doi. org/10.4028/www.scientific.net/MSF.893.57

López, L., Sarmiento, A., Fajardo, J., Valarezo, L., y Zuluaga, R. (s. f.). Determinación del porcentaje de humedad, solubles e insolubles en agua de la fibra de Carludovica Palmata (paja toquilla).

Ma, Y., Ueda, M., Yokozeki, T., Sugahara, T., Yang, Y., y Hamada, H. (2017). A comparative study of the mechanical properties and failure behavior of carbon fiber/epoxy and carbon fiber/polyamide 6 unidirectional composites. Composite Structures, 160 (Supplement C), 89-99. https://doi. org/10.1016/j.compstruct.2016.10.037

Ma, Y., Yang, Y., Sugahara, T., y Hamada, H. (2016). A study on the failure behavior and mechanical properties of unidirectional fiber reinforced thermosetting and thermoplastic composites. Composites Part B: Engineering, 99: 162-172. https://doi.org/10.1016/j.compositesb.2016.06.005

Majerski, K., Surowska, B., y Bieniaś, J. (2012). Tensile properties of carbon fiber/epoxy laminates at low and room temperatures. Composites Theory and Practice, R. 12 (3): 182-185. 
Mallick, P. K. (2007). Fiber-Reinforced Composites: Materials, Manufacturing, and Design, Third Edition. CRC Press.

Mcllhagger, A., Archer, E., y Mcllhagger, R. (2015). 3 - Manufacturing processes for composite materials and components for aerospace applications. En P. E. Irving y C. Soutis (eds.), Polymer Composites in the Aerospace Industry (53-75). https://doi.org/10.1016/B978-0-85709-523-7.00003-7

Mehta, M. G., y Vadher, J. A. (2017). A study on Different Failures of Composite Materials. International Journal of Advance Engineering and Research Development, 4 (9), 302-307.

Murugan, R., Ramesh, R., y Padmanabhan, K. (2014). Investigation on Static and Dynamic Mechanical Properties of Epoxy Based Woven Fabric Glass/Carbon Hybrid Composite Laminates. Procedia Engineering, 97: 459-468. https://doi.org/10.1016/j.proeng.2014.12.270

Noroña, M., y Gómez, M. (2019). Desarrollo e innovación de los sistemas mecatrónicos en un automóvil: Una revisión. Enfoque UTE, 10 (1): 117-127. https://doi.org/10.29019/enfoqueute.v10n1.350

Paiva, J. M. F. de, Mayer, S., \& Rezende, M. C. (2006). Comparison of tensile strength of different carbon fabric reinforced epoxy composites. Materials Research, 9 (1): 83-90. https://doi.org/10.1590/ S1516-14392006000100016

Paltán, C. (2016). Validación del sistema de inyección de un biomaterial compuesto PP-GAK mediante modelamiento CAD-CAE. Universidad Politécnica Salesiana.

Paredes, J., Pérez, C., y Castro, C. (2017). Análisis de las propiedades mecánicas del compuesto de matriz poliéster reforzado con fibra de vidrio 375 y cabuya aplicado a la industria automotriz. Enfoque UTE, 8 (3), 1-15. https://doi.org/10.29019/enfoqueute.v8n3.163

Ramana, M. V., y Ramprasad, S. (2017). Experimental Investigation on Jute/Carbon Fibre reinforced Epoxy based Hybrid Composites. Materials Today: Proceedings, 4 (8): 8654-8664. https://doi. org/10.1016/j.matpr.2017.07.214

Schulenberg, L., Seelig, T., Andrieux, F., y Sun, D.-Z. (2017). An anisotropic elasto-plastic material model for injection-molded long fiber-reinforced thermoplastics accounting for local fiber orientation distributions. Journal of Composite Materials, 51 (14): 2061-2078. https://doi. org/10.1177/0021998316668983

Viscardi, M., Arena, M., Barra, G., y Guadagno, L. (2016). Structural performance analysis of smart carbon fiber samples supported by experimental investigation. International Journal of Mechanics, 10, 376-382.

Wang, K., Zhao, L., Hong, H., Gong, Y., Zhang, J., \& Hu, N. (2019). An analytical model for evaluating the buckling, delamination propagation, and failure behaviors of delaminated composites under uniaxial compression. Composite Structures, 223. https://doi.org/10.1016/j.compstruct.2019.110937

Yaqiang, Y., Xin, W., y Zhishen, W. (2017). Damping Behavior of Hybrid Fiber-Reinforced Polymer Cable with Self-Damping for Long-Span Bridges. Journal of Bridge Engineering, 22 (7): 05017005. https:// doi.org/10.1061/(ASCE)BE.1943-5592.0001058 